\section{Kidney \\ Blood Pressure \\ Research}

\title{
Revealing the Underlying Mechanism of Ischemia Reperfusion Injury Using Bioinformatics Approach
}

\author{
Bingbing Shen Shan Zhou Yue He Hongwen Zhao Mei Mei Xiongfei Wu \\ Department of kidney, Southwest hospital of Third Military Medical University, Chongqing City, 400038, \\ China
}

\section{Key Words}

Ischemia reperfusion injury • Differentially expressed genes $•$ Protein-protein interaction network $•$ MicroRNA-target gene network $•$ Functional analysis

\begin{abstract}
Background/Aims: To reveal the potential pathogenesis of ischemia/reperfusion (I/R) injury. Methods: GSE9943 were downloaded from Genome Expression Omnibus database, including I/R and control samples for both Brown Norway (BN) and Sprague Dawley (SD) rats (3 rats/each group). Then differentially expressed genes (DEGs) were identified by limma package. miRNAtarget gene network pairs were predicted using WebGestalt, and protein-protein interactions (PPI) were identified based on STRING database, followed by the networks construction using Cytoscape. Next, ClusterONE was used for modules screening. Furthermore, functional analyses were performed to common DEGs and genes. Results: Totally, 23 common DEGs of $B R$ and SD rats were screened, enriched in functions, such as regulation of cellular protein metabolic process, response to wounding, proteinaceous extracellular matrix, and Enzyme inhibitor activity. MIR-29A, MIR-29B and MIR-29C were discovered both in up- and downregulated miRNA-target gene networks. Genes in the PPI network were significantly disturbed in p53 signaling, complement and coagulation cascades pathway. Four modules were found significantly disturbed cytochrome P450, Serine/threonine protein kinase, calcium binding and Transient receptor potential channel protein domains. Conclusion: During I/R injury, many genes mutated, interrupting several biological functions, pathways and protein domains. MIR$29 \mathrm{C}$ and TRPC6 were suggested to be potential novel targets for this disease.
\end{abstract}

Copyright $@ 2014$ S. Karger AG, Basel

\section{Introduction}

The first ischemia reperfusion injury (I/R) was recognized in 1970s and the term indicates tissue ischemia with inadequate oxygen supply followed by successful reperfusion 


\section{Kidney \\ Blood Pressure Research}

Kidney Blood Press Res 2013;38:99-108

\begin{tabular}{l|l}
\hline DOI: 10.1159/000355759 & C 2014 S. Karger AG, Basel
\end{tabular}

Publisned online: February 26, 2014

www.karger.com/kbr

Shen/Zhou/He/Zhao/Mei/Wu: The Mechanism of I/R Injury

initiating a wide and complex array of inflammation responses [1]. Due to the severity of this disease, numbers of researchers have been engaging in revealing the potential pathogenesis. M Kawaguchi et al. discover that inflammation activation of cardiac fibroblasts is essential for myocardial ischemia/reperfusion injury [2]. Toll-like receptor-mediated signaling pathways was implicated in ischemia I/R [3]. Although several factors associated with I/R have been discovered, the mechanism of this disease is not fully indicated. Therefore, new targets needed to be discovered.

MicroRNAs (miRNAs) are integral elements in the post-transcriptional control of gene expression [4] and these small molecules have the potential to influence cellular behavior [5]. During last few decades, several miRNAs have been found playing roles in I/R development. For example: miRNA-320 is involved in the regulation of cardiac ischemia I/R by targeting heat-shock protein 20 [6]. miRNA-494 is down-regulated in murine I/R-injured and human infarcted hearts [7]. MiRNAs are regulators of target genes and miRNA-target gene pairs play important roles in biological behaviors. miR-124 is identified to be an endogenous regulator of Ku70 that improves I/R-induced brain injury [8]. Thus, in our study, the roles of miRNAtarget gene pairs in I/R development were investigated.

Brown Norway (BN) rats strain was discovered to be dramatically resistant to cardiac and renal I/R injury compared with other strains, while Sprague Dawley (SD) rats are more susceptible with I/R injury [9]. Resistance to the development of tubular damage after renal ischemia was also observed in BN rats, and higher levels of heat-shock proteins and ablated oxidative stress was believed to be the putative mechanism of this resistance $[10,11]$. BN rats strain is also reported to be an useful experimental model to investigate the mechanism of I/R injury compared with SD rats strain [12]. Despite of all the previously reported works, the genetic response of these two kinds of rats to ischemic insults was not clearly elucidated.

In the present research, the differentially expressed genes (DEGs) between I/R and control rats (BN and SD) were selected and their functions were analyzed. Additionally, the miRNA-target gene relationships and protein-protein interactions (PPIs) were predicted and the corresponding networks were constructed. Furthermore, the potential mechanism of I/R was revealed by clustering and functional analysis for PPI network. Based on the findings, the pathogenesis of I/R was investigated and several potential novel targets were discovered for this disease.

\section{Materials and Methods}

\section{Data extracting and sampling}

The data GSE9943 [13] were downloaded from the National Center for Biotechnology Information (NCBI) Genome Expression Omnibus (GE0, http://www.ncbi.nlm.nih.gov/geo/) database [14]. Totally 12 samples were available within the data, including two groups (I/R group and control group) for both Brown Norway (BN) and Sprague Dawley (SD) rats ( $\mathrm{n}=3$ per group). Gene annotation information was also downloaded from the platform of GPL 2996 ABI Rat Genome Survey Microarray, which contained 26857 probes.

\section{Data processing and DEGs identifying}

The rows containing missing values were deleted from the original file, and then one file included 12925 rows and 12 columns was obtained. Next, limma package [15] in R (V.3.0.1) [16] was used to identify the DEGs between I/R group and control group in the two rat strains. Bayes testing [17] was applied to perform the multiple correction, and p values $<0.05$ and $\mid \log$ fold change (FC) $\mid>1.0$ were set as the threshold.

\section{Functional enrichment analysis}

DAVID (Database for Annotation, Visualization and Integrated Discovery [18]) was utilized to perform protein-domains, Gene Ontology (GO) [19] and pathway enrichment analyses based on hypergeometric analysis. The databases InterPro [20] and KEGG (kyoto encyclopedia of genes and genomes) [21] were used for protein-domains and pathway predication, respectively. P values less than 0.05 were set as the threshold. 


\section{Kidney Blood Pressure Research}

MiRNA-target gene network

MicroRNA Target Analysis tool in WebGestalt (WEB-based GEne SeT AnaLysis Toolkit) [22] was used to predict the miRNAs for up- and downregulated genes. miRNAs with multiple test adjustment $\mathrm{p}$ values (AdjP) less than 0.1 were selected. Then the miRNA-target gene network was constructed using Cytoscape [23].

Protein-protein interactions (PPI) network constructing

It has been discovered that functional links between proteins can be inferred from genomic associations between the genes that encode them, and the genes are often located in close proximity on the genome. STRING database (http://www.bork. embl-heidelberg.de/STRING/] [24] is a precomputed global resource for the exploration and analysis of these associations. STRING is updated continuously and currently, and now contains 261033 orthologs in 89 fully sequenced genomes. In the present study, STRING 9.0 was used to screen the interactions among the DEGs, and the PPIs with combined scores more than 0.4 were selected for PPI network construction. What's more, pathway annotation was conducted by DAVID with p value less than 0.05 .

Module analysis of the PPI network

The Clustering analysis of the PPI network was performed using the ClusterONE in Cytoscape [25] with the minimum size as 5 and minimum density as 0.05 , to select the functional modules. P values less than 1.0E-5 were selected as threshold. Moreover, protein domain enrichment analysis of the modules was performed by DAVID and p values less than 0.05 were set as threshold.

\section{Results}

The identified DEGs

Using limma package, the DEGs between I/R and control BN and SD rats were identified. Finally, 92 DEGs were screened in BN rats, including 69 up-regulated and 23 down-regulated genes. Meanwhile, 176 DEGs were discovered in SD rats which contained 92 up-regulated and 84 down-regulated genes. Interestingly, 23 DEGs were found both in BN and SD rats, including up-regulated genes: Angpt14, Atp12a, Ccnb1, Emp3, Fenl, Foxml, and Gpnmb; down-regulated genes: Ces1,Cox8h, Gdf15, and Gsta5 (Figure 1).

\section{Functional analysis of common DEGs}

Further, GO analysis was performed to the 23 common DEGs and the significant terms with p values less than 0.05 were selected (Table 1 ). The common DEGs were discovered significantly enriched in 8 biological process related GO terms, including the regulation of cellular protein metabolic process $(p=0.022)$, response to wounding $(p=0.023)$, and $M$ phase $(\mathrm{p}=0.027)$. Totally 4 cellular component related terms were enriched, such as proteinaceous extracellular matrix $(p=0.005)$ and extracellular matrix $(p=0.007)$. Enzyme inhibitor activity was the only molecular function related term $(p=0.039)$.

\section{MiRNA-target gene network}

Based on the 245 DEGs between I/R and control rats, which included 142 up-regulated and 103 down-regulated genes, the corresponding miRNAs were predicted. Significant miRNA-target gene pairs were selected with AdjP $<0.1$ and the miRNA-target gene network 


\section{Kidney \\ Blood Pressure Research}

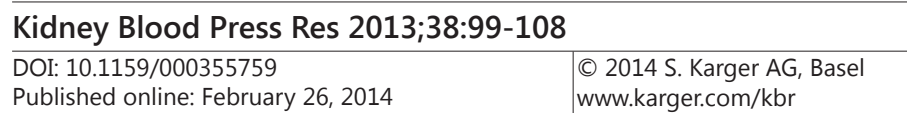

C) 2014 S. Karger AG,
www.karger.com/kbr

Table 1. The significant GO terms of the 23 common DEGs with P Values $<0.05$

\begin{tabular}{lllcl}
\hline Category & Term & Description & Count & P Value \\
\hline BP & GO:0032268 & regulation of cellular protein metabolic process & 4 & 0.022461188 \\
BP & GO:0009611 & response to wounding & 4 & 0.023608951 \\
BP & GO:0000279 & M phase & 3 & 0.027081944 \\
BP & GO:0042060 & wound healing & 3 & 0.027081944 \\
BP & GO:0007049 & cell cycle & 4 & 0.029170792 \\
BP & GO:0040007 & growth & 3 & 0.039937324 \\
BP & GO:0045861 & negative regulation of proteolysis & 2 & 0.040898315 \\
BP & GO:0022403 & cell cycle phase & 3 & 0.046190442 \\
CC & GO:0005578 & proteinaceous extracellular matrix & 4 & 0.004619661 \\
CC & GO:0031012 & extracellular matrix & 4 & 0.006741095 \\
CC & GO:0044421 & extracellular region part & 5 & 0.020594376 \\
CC & GO:0005576 & extracellular region & 6 & 0.042916516 \\
MF & GO:0004857 & enzyme inhibitor activity & 3 & 0.039374025 \\
\hline
\end{tabular}

Note: BP, CC and MF are biological process, cellular component and molecular function, respectively. GO represents the gene ontology. Count is the DEGs enriched in the corresponding GO term. DEGs: differentially expressed genes.

was established by Cytoscape (Figure 2).

MiRNA-target gene network of up-regulated genes (Figure 2A), included 39 nodes and 77 edges. Genes, Dll1, Bdnf and Gmfb were discovered with the highest degree of 10,9 and 9 respectively in this network. Meanwhile, MIR-29A, MIR-29B and MIR-29C were found regulated the most target genes (5), such as Slc16al, Gmfb, Gpr37, Dnm3 and Fbn1.

MiRNA-target gene network of down-regulated genes contained 29 nodes and 35 edges. Igf1 and Nptx1 had the highest degrees of 7 and 4, respectively. Interestingly, the miRNAs which regulated most target genes were also MIR-29A, MIR-29B and MIR29C. These three miRNAs regulated Igf1, Trim63, Gpr37 and Prelp in the network.

\section{PPI network}

The PPIs among 245 DEGs were predicted by SRTING and the significant ones with combined score $>0.4$ were selected to construct the PPI network. The PPI is shown in

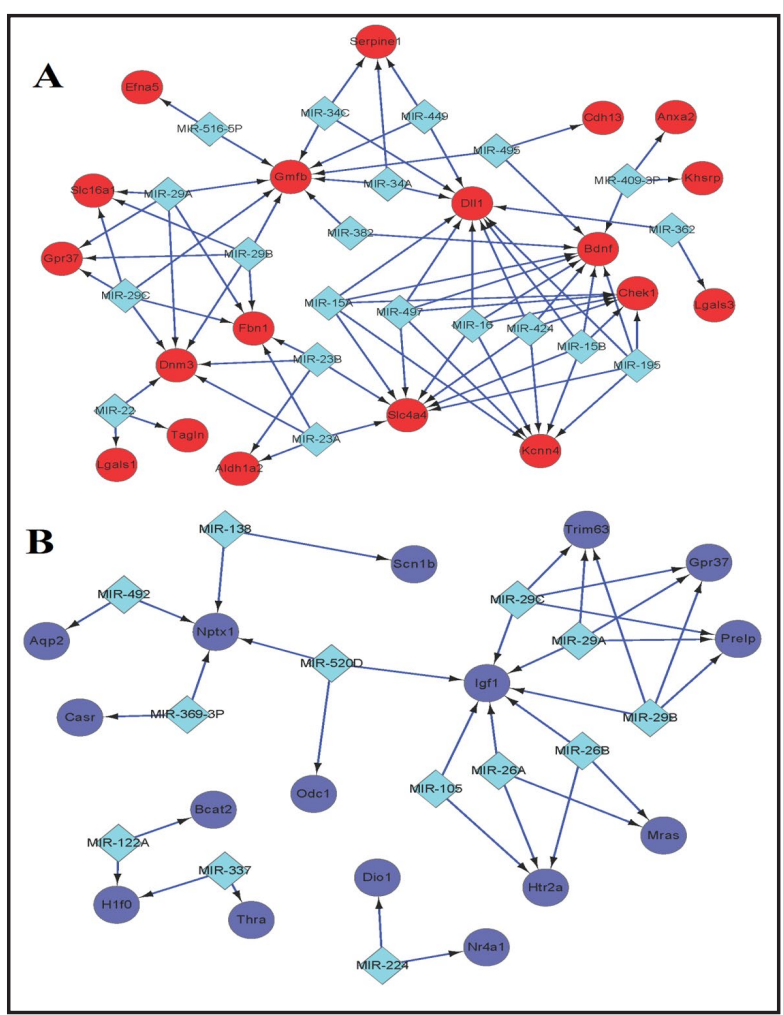

Fig. 2. The miRNA-target gene network. A and B represent the up- and down-regulated network, respectively. The diamond nodes are miRNAs. The red and blue round nodes are up- and down-regulated target genes, respectively.

Figure 3, containing 112 nodes and 228 edges. Genes which had the highest degrees included Agt $($ Degree $=16)$, Timp1 $($ Degree $=15)$, Egfr $($ Degree $=15)$, Cat $($ Degree $=15)$, and Top2a (Degree $=13$ ). 


\section{Kidney \\ Blood Pressure Research}

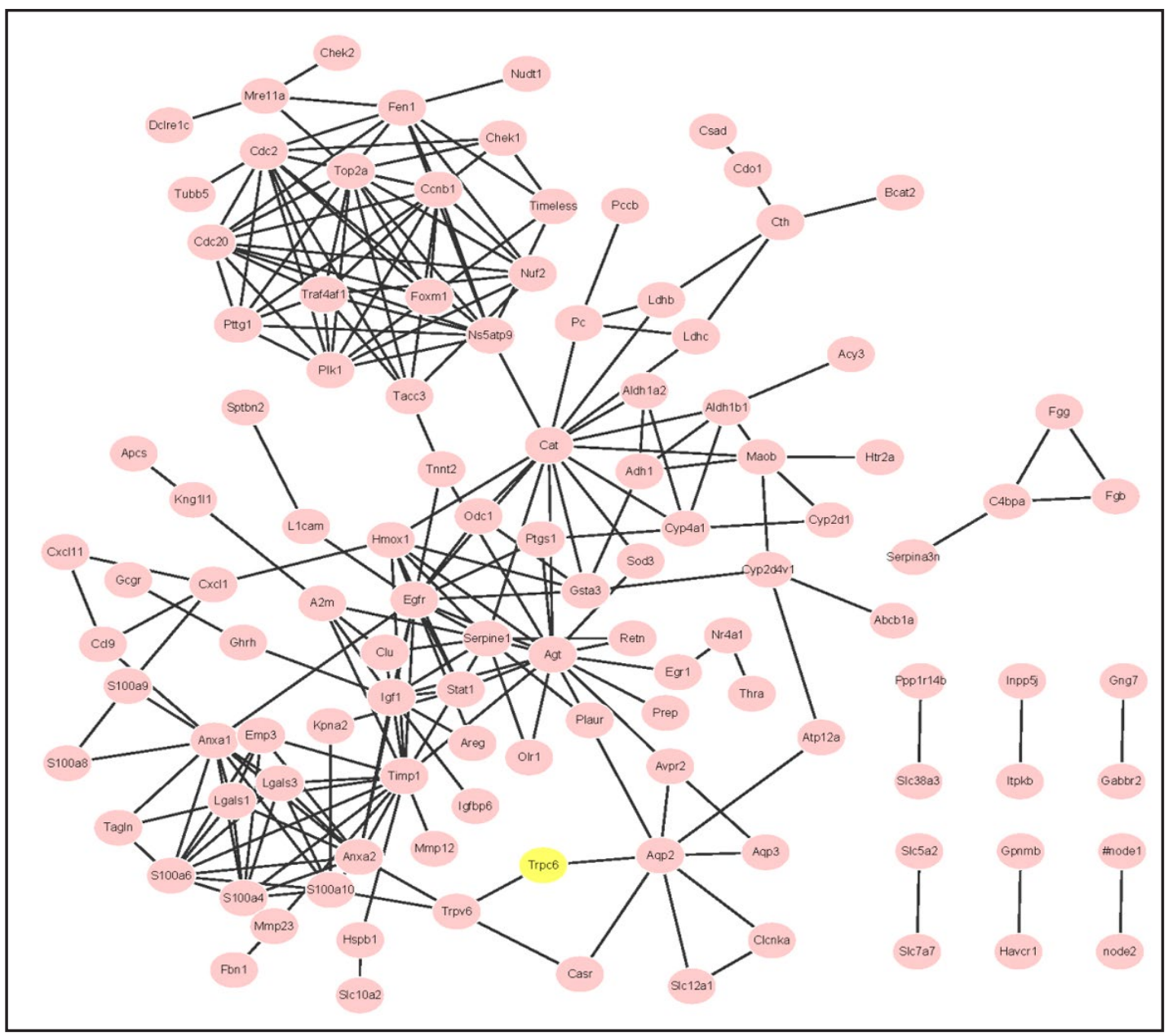

Fig. 3. The PPI network for DEGs between I/R and control rats. The nodes and edges represent the proteins (genes) and interactions, respectively. The thick edges stand for the large scores, indicating the predicted results are precise. Yellow node is the TRPC6 gene.

\section{Pathway enrichment analysis for PPI network}

Moreover, pathway enrichment analysis was conducted to the genes in the PPI network using DAVID and the significant pathways with p values $<0.05$ were listed in Table 2 . The most significant pathways were $\mathrm{p} 53$ signaling pathway $[26,27](\mathrm{p}=4.72 \mathrm{E}-04)$ and complement and coagulation cascades [28] $(\mathrm{p}=6.20 \mathrm{E}-04)$. Meanwhile, the DEGs enriched in these two pathways included CCNB1, CDC2, SERPINE1, A2M and FGG.

\section{Module analysis}

Four modules, Module 1 (Nodes $=35$, Density $=0.119$, Quality $=0.747$, P-value $=1.019 \mathrm{E}-9$ ), Module 2 (Nodes $=14$, Density $=0.615$, Quality $=0.903$, P-value $=2.459 \mathrm{E}-6)$, Module 3 (Nodes $=12$, Density $=0.530$, Quality $=0.673$, P-value $=5.715 \mathrm{E}-4$ ), and Module 4 $($ Nodes $=11$, Density $=0.236$, Quality $=0.565$, P-value $=1.0 \mathrm{E}-3)$ were obtained during PPI clustering analysis by using ClusterONE in Cytoscape and the result is shown in Figure 4.

Furthermore, we conducted protein domain enrichment analysis of the genes in the four Modules by DAVID, and the significant protein domains with $\mathrm{p}$ values $<0.05$ are listed in Table 3. The protein domains in Module 1 significantly enriched in cytochrome P450 (p $=1.19 \mathrm{E}-2$ ) and the genes included CYP4A1, CYP2D4V1, and CYP2D1. The protein domains in Module 2 significantly enriched in Serine/threonine protein kinase $(p=7.84 \mathrm{E}-3)$ and 


\section{Kidney Blood Pressure Research}

\section{Kidney Blood Press Res 2013;38:99-108}

\begin{tabular}{l|l}
\hline DOI: $10.1159 / 000355759$ & C 2014 S. Karger AG, Basel
\end{tabular}

Publisned online: February 26, 2014

www.karger.com/kbr

Table 2. The significant pathways in PPI network with $\mathrm{p}$ values $<0.05$

\begin{tabular}{llcll}
\hline Term & Description & Count & Genes & P Value \\
\hline rno04115 & p53 signaling pathway & 6 & $\begin{array}{l}\text { CCNB1, CDC2, SERPINE1, } \\
\text { IGF1, CHEK1, CHEK2 }\end{array}$ & $4.72 E-04$ \\
rno04610 & Complement and coagulation cascades & 6 & $\begin{array}{l}\text { A2M, FGG, FGB, SERPINE1, } \\
\text { C4BPA, PLAUR }\end{array}$ & $6.20 E-04$ \\
rno04110 & Cell cycle & 7 & $\begin{array}{l}\text { CCNB1, PLK1, CDC2, CDC20, } \\
\text { CHEK1, PTTG1, CHEK2 }\end{array}$ & 0.001502 \\
rno04114 & Oocyte meiosis & 6 & $\begin{array}{l}\text { CCNB1, PLK1, CDC2, IGF1, } \\
\text { CDC20, PTTG1 }\end{array}$ & 0.004815 \\
rno00270 & Cysteine and methionine metabolism & 4 & LDHC, LDHB, CTH, CD01 & 0.004968 \\
rno00982 & Drug metabolism & 5 & $\begin{array}{l}\text { GSTA3, ADH1, MAOB, } \\
\text { CYP2D4V1, CYP2D1 }\end{array}$ & 0.00561 \\
rno03450 & Non-homologous end-joining & 3 & DCLRE1C, MRE11A, FEN1 & 0.007164 \\
rno00640 & Propanoate metabolism & 3 & LDHC, LDHB, PCCB & 0.04269 \\
rno04540 & Gap junction & 4 & EGFR, CDC2, TUBB5, HTR2A & 0.046936 \\
\hline
\end{tabular}

Count is the DEGs enriched in the corresponding pathways.

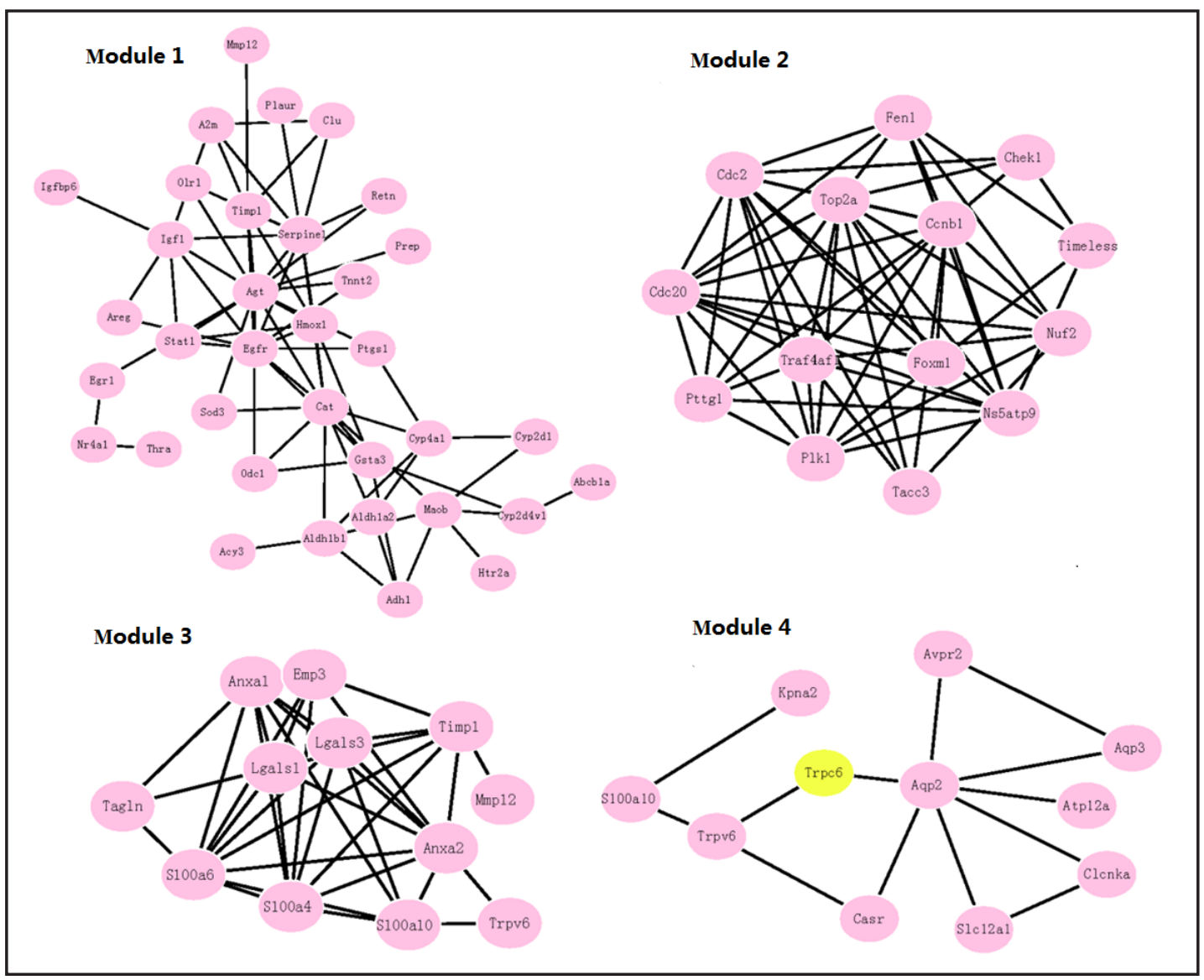

Fig. 4. Modules in PPI network. There are 35, 14, 12, and 11 nodes in Module 1, 2, 3 and 4, respectively.

the genes included PLK1, CDC2, and CHEK1. The protein domains in Module 3 significantly enriched in calcium binding ( $\mathrm{p}=1.48 \mathrm{E}-4)$ and the genes included S100A4, S100A6, and S100A10. The protein domains in Module 4 significantly enriched in Transient receptor potential channel $(\mathrm{p}=1.48 \mathrm{E}-4)$ and the genes included TRPC6, and TRPV6. 


\section{Kidney \\ Blood Pressure Research}

Kidney Blood Press Res 2013;38:99-108

DOI: $10.1159 / 000355759$

Published onlıne: February 26, 2014

(C) 2014 S. Karger AG, Base

www.karger.com/kbr

Table 3. The significant protein domains of genes in four modules with $p<0.05$

\begin{tabular}{|c|c|c|c|c|}
\hline Term & Description & Count & PValue & Genes \\
\hline \multicolumn{5}{|l|}{ Module 1} \\
\hline IPR002401 & Cytochrome P450, E-class, group I & 3 & $1.19 \mathrm{E}-02$ & CYP4A1, CYP2D4V1, CYP2D1 \\
\hline IPR017973 & Cytochrome P450, C-terminal region & 3 & $1.95 \mathrm{E}-02$ & CYP4A1, CYP2D4V1, CYP2D1 \\
\hline IPR008069 & $\begin{array}{l}\text { Cytochrome P450, E-class, group I, } \\
\text { CYP2D-like }\end{array}$ & 2 & $1.98 \mathrm{E}-02$ & CYP2D4V1, CYP2D1 \\
\hline IPR017972 & Cytochrome P450, conserved site & 3 & $2.01 \mathrm{E}-02$ & CYP4A1, CYP2D4V1, CYP2D1 \\
\hline IPR001128 & Cytochrome P450 & 3 & $2.13 \mathrm{E}-02$ & CYP4A1, CYP2D4V1, CYP2D1 \\
\hline \multicolumn{5}{|l|}{ Module 2} \\
\hline IPR002290 & Serine/threonine protein kinase & 3 & $7.84 \mathrm{E}-03$ & PLK1, CDC2, CHEK1 \\
\hline IPR008271 & Serine/threonine protein kinase, active site & 3 & $1.40 \mathrm{E}-02$ & PLK1, CDC2, CHEK1 \\
\hline IPR017442 & Serine/threonine protein kinase-related & 3 & $1.71 \mathrm{E}-02$ & PLK1, CDC2, CHEK1 \\
\hline IPR017441 & Protein kinase, ATP binding site & 3 & $2.27 \mathrm{E}-02$ & PLK1, CDC2, CHEK1 \\
\hline IPR000719 & Protein kinase, core & 3 & $2.89 \mathrm{E}-02$ & PLK1, CDC2, CHEK1 \\
\hline \multicolumn{5}{|l|}{ Module 3} \\
\hline IPR013787 & $\begin{array}{l}\text { S100/CaBP-9k-type, calcium binding, } \\
\text { subdomain }\end{array}$ & 3 & $1.38 \mathrm{E}-04$ & S100A4, S100A6, S100A10 \\
\hline IPR001751 & S100/CaBP-9k-type, calcium binding & 3 & $1.48 \mathrm{E}-04$ & S100A4, S100A6, S100A10 \\
\hline IPR001464 & Annexin & 2 & $9.21 \mathrm{E}-03$ & ANXA1, ANXA2 \\
\hline IPR018252 & Annexin repeat, conserved site & 2 & $9.21 \mathrm{E}-03$ & ANXA1, ANXA2 \\
\hline IPR018502 & Annexin repeat & 2 & $9.21 \mathrm{E}-03$ & ANXA1, ANXA2 \\
\hline IPR001079 & Galectin, carbohydrate recognition domain & 2 & $9.86 \mathrm{E}-03$ & LGALS3, LGALS1 \\
\hline IPR011992 & EF-Hand type & 3 & $1.01 \mathrm{E}-02$ & S100A4, S100A6, S100A10 \\
\hline IPR013320 & $\begin{array}{l}\text { Concanavalin A-like lectin/glucanase, } \\
\text { subgroup }\end{array}$ & 2 & 4.97E-02 & LGALS3, LGALS1 \\
\hline \multicolumn{5}{|l|}{ Module 4} \\
\hline IPR004729 & Transient receptor potential channel & 2 & $6.59 \mathrm{E}-03$ & TRPC6, TRPV6 \\
\hline IPR012269 & Aquaporin & 2 & $6.59 \mathrm{E}-03$ & AQP3, AQP2 \\
\hline IPR000425 & Major intrinsic protein & 2 & 8.97E-03 & AQP3, AQP2 \\
\hline
\end{tabular}

\section{Discussion}

In the present research, 23 common DEGs both in BR and SD rats were identified and these genes were found significantly interrupted several functions related with biological processes, cellular component and molecular function, including regulation of cellular protein metabolic process, response to wounding, $\mathrm{M}$ phase and wound healing. In addition, based on miRNA-target gene network, MIR-29A, MIR-29B and MIR-29C were discovered played important roles in I/R. Moreover, Agt, Timp1, Egfr, Cat, Cdc2, and Top2a were found to have the highest degrees in the PPI network and the genes mainly enriched in p53 signaling pathway and complement and coagulation cascades pathway. Finally, four modules in the PPI network were screened out, and they were significantly enriched in protein domains of cytochrome P450, Serine/threonine protein kinase, calcium binding and Transient receptor potential channel, respectively.

The relationships between miRNAs and target genes were predicted and two miRNAtarget gene networks, including up- and down-regulated networks were constructed. Both in these two networks, MIR-29A, MIR-29B and MIR-29C were selected as they had the highest degrees, indicating these miRNAs may play important roles in disease development. Ouyang, Y.B., et al have reported that astrocyte-enriched miR-29a targets PUMA and reduces neuronal vulnerability to forebrain ischemia [29]. Additionally, Mateo Ziu et al. have found that expression of miR-29b is significantly decreased in neurons exposed to insulin-like 


\section{Kidney \\ Blood Pressure Research}

Kidney Blood Press Res 2013;38:99-108

\begin{tabular}{l|l}
\hline DOI: $10.1159 / 000355759$ & (C) 2014 S. Karger AG, Basel
\end{tabular}

Published online: February 26, 2014

www.karger.com/kbr

Shen/Zhou/He/Zhao/Mei/Wu: The Mechanism of I/R Injury

growth factor 1, a well documented neuroprotectant in ischemic models [30]. Our results are consistent with these previous findings. On the other hand, miR-29c was also discovered in our study but with no previous evidence. S Sengupta et al. have shown that miR-29c is expressed at one-fifth the levels in tumors as in normal epithelium [31]. Other researchers discover that miR-29c targets TNFAIP3, inhibits cell proliferation and induces apoptosis in hepatitis B virus-related hepatocellular carcinoma [32]. In our study, miR-29c was found connected with five target genes in up- and down-regulated networks, suggesting this may be a novel target for I/R research.

The established PPI network included 112 nodes and 228 edges. Angiotensinogen (Agt), tissue inhibitor of metalloproteinases-1 (Timp1), the epidermal growth factor receptor (Egfr), chloramphenicol acetyltransferase (Cat), (Csc2), and topoisomerase IIa (Top2a) were discovered to be connected with more than 12 genes in the network, indicating these genes may be involved in the disease developing. Further, these 112 nodes in PPI network were significantly enriched in p53 signaling pathway and Complement and coagulation cascades pathways. The p53 tumor-suppressor plays a critical role in the prevention of human cancer, and in response to various types of stress p53 becomes activated, cells can undergo marked phenotypic changes, ranging from increased DNA repair to senescence and apoptosis [33]. Thus, in our findings, genes may interrupt these two pathways to induce I/R.

What's more, given that PPI network was too large to be analyzed, we conducted clustering analysis and screened out four modules, followed by the protein domains enrichment. The protein domains in Module 4 significantly enriched in Transient receptor potential channel, and canonicaltransient receptor potential channel 6 (TRPC6) was among the genes in this module. TRPC6 have been reported to be associated with I/R in the lung [34], brain I/R injury is reported to be prevented through TRPC6-MEK-CREB and TRPC6CaMKIV-CREB pathways [35], and activating TRPC channels before ischemia is believed to have early neuroprotective effects on retinal ganglion cells in vitro [36]. In this study, log FC for TRPC6 between normal and I/R injury rats samples was 1.53, indicating that TRPC6 was an up-regulated DEG. Currently, there have been no reports showing the relations between I/R injury in kidney and TRPC6, therefore, our finding may provide new evidence.

Only 6 samples were available for each rat strain in this bioinformatics analysis. The sample size is relative small, thus, further analysis based on a larger sample size should be performed.

\section{Conclusion}

The potential mechanism of I/R injury in kidney in rats model was investigated in our present research. Our findings suggested that several GO terms, pathways and protein domains may be interrupted during the disease progress. Meanwhile, miR-29c and TRPC6 were suggested to be potential novel targets for this disease. Our study revealed the underlying mechanism of I/R from several levels and obtained some new results. These findings have the potential to investigate the pathogenesis of I/R injury.

\section{Conflict of Interests}

All authors declare that they have no conflict of interest. 


\section{Kidney \\ Blood Pressure Research}

Kidney Blood Press Res 2013;38:99-108

\begin{tabular}{l|l}
\hline DOI: 10.1159/000355759 & (C) 2014 S. Karger AG, Basel
\end{tabular}

Published online: February 26, 2014

www.karger.com/kbr

Shen/Zhou/He/Zhao/Mei/Wu: The Mechanism of I/R Injury

\section{Reference}

1 Dorweiler B, Pruefer D, Andrasi TB, Maksan SM, Schmiedt W, Neufang A, Vahl CF: Ischemia-reperfusion injury. Eur J Trauma Emerg Surg 2007;33:600-612.

-2 Kawaguchi M, Takahashi M, Hata T, Kashima Y, Usui F, Morimoto H, Izawa A, Takahashi Y, Masumoto J, Koyama J: Inflammasome activation of cardiac fibroblasts is essential for myocardial ischemia/reperfusion injury. Circulation 2011;123:594-604.

- Ha T, Hu Y, Liu L, Lu C, McMullen JR, Kelley J, Kao RL, Williams DL, Gao X, Li C: TLR2 ligands induce cardioprotection against ischaemia/reperfusion injury through a PI3K/Akt-dependent mechanism. Cardiovasc Res 2010;87:694-703.

-4 Inui M, Martello G, Piccolo S: MicroRNA control of signal transduction. Nat Rev Mol Cell Biol 2010;11:252263.

5 Kota J, Chivukula RR, O'Donnell KA, Wentzel EA, Montgomery CL, Hwang H-W, Chang T-C, Vivekanandan P, Torbenson M, Clark KR: Therapeutic microRNA delivery suppresses tumorigenesis in a murine liver cancer model. Cell 2009;137:1005-1017.

6 Ren X-P, Wu J, Wang X, Sartor MA, Qian J, Jones K, Nicolaou P, Pritchard TJ, Fan G-C: MicroRNA-320 is involved in the regulation of cardiac ischemia/reperfusion injury by targeting heat-shock protein 20. Circulation 2009;119:2357-2366.

7 Wang X, Zhang X, Ren X-P, Chen J, Liu H, Yang J, Medvedovic M, Hu Z, Fan G-C: MicroRNA-494 targeting both proapoptotic and antiapoptotic proteins protects against ischemia/reperfusion-induced cardiac injury. Circulation 2010;122:1308-1318.

8 Zhu F, Liu J-L, Li J-P, Xiao F, Zhang Z-X, Zhang L: MicroRNA-124 (miR-124) Regulates Ku70 Expression and is Correlated with Neuronal Death Induced by Ischemia/Reperfusion. J Mol Neurosci 2013;1-8.

-9 Nilakantan V, Hilton G, Maenpaa C, Van Why SK, Pieper GM, Johnson CP, Shames BD: Favorable balance of anti-oxidant/pro-oxidant systems and ablated oxidative stress in Brown Norway rats in renal ischemiareperfusion injury. Mol Cell Biochem 2007;304:1-11.

10 Shi Y, Hutchins W, Ogawa H, Chang C-C, Pritchard KA, Zhang C, Khampang P, Lazar J, Jacob HJ, Rafiee P: Increased resistance to myocardial ischemia in the Brown Norway vs. Dahl S rat: role of nitric oxide synthase and Hsp90. J Mol Cell Cardiol 2005;38:625-635.

11 Baker JE, Konorev EA, Gross GJ, Chilian WM, Jacob HJ: Resistance to myocardial ischemia in five rat strains: is there a genetic component of cardioprotection? Am J Physiol Heart Circ Physiol 2000;278:H1395-H1400.

12 Sáenz-Morales D, Conde E, Blanco-Sánchez I, Ponte B, Aguado-Fraile E, de las Casas G, García-Martos M, Alegre L, Escribese MM, Molina A: Differential resolution of inflammation and recovery after renal ischemia-reperfusion injury in Brown Norway compared with Sprague Dawley rats. Kidney Int 2010;77:781-793.

13 Viñas JL, Sola A, Jung M, Mastora C, Vinuesa E, Pi F, Hotter G: Inhibitory action of Wnt target gene osteopontin on mitochondrial cytochrome c release determines renal ischemic resistance. Am J Physiol Renal Physiol 2010;299:F234-F242.

14 Barrett T, Troup DB, Wilhite SE, Ledoux P, Rudnev D, Evangelista C, Kim IF, Soboleva A, Tomashevsky M, Edgar R: NCBI GEO: mining tens of millions of expression profiles--database and tools update. Nucleic Acids Res 2007;35:D760-765.

-15 Diboun I, Wernisch L, Orengo C, Koltzenburg M: Microarray analysis after RNA amplification can detect pronounced differences in gene expression using limma. BMC genomics 2006;7:252.

16 Team RDC: R: A language and environment for statistical computing. R Foundation for Statistical Computing. Vienna, Austria 2005; In: ISBN 3-900051-07-0. (url: http://www. R-project. org): 2013.

17 Dudoit S, Gilbert HN, Van Der Laan MJ: Resampling-based empirical Bayes multiple testing procedures for controlling generalized tail probability and expected value error rates: focus on the false discovery rate and simulation study. Biom J 2008;50:716-744.

18 Da Wei Huang BTS, Lempicki RA: Systematic and integrative analysis of large gene lists using DAVID bioinformatics resources. Nat Protoc 2008;4:44-57.

19 Ashburner M, Ball CA, Blake JA, Botstein D, Butler H, Cherry JM, Davis AP, Dolinski K, Dwight SS, Eppig JT, Harris MA, Hill DP, Issel-Tarver L, Kasarskis A, Lewis S, Matese JC, Richardson JE, Ringwald M, Rubin GM, Sherlock G: Gene ontology: tool for the unification of biology. The Gene Ontology Consortium. Nat Genet 2000;25:25-29. 


\section{Kidney \\ Blood Pressure Research}

Kidney Blood Press Res 2013;38:99-108

\begin{tabular}{l|l|}
\hline DOI: $10.1159 / 000355759$ & (C) 2014 S. Karger AG, Basel
\end{tabular}

Publisned online: February 26, 2014

www.karger.com/kbr

20 Mulder NJ, Apweiler R: The InterPro database and tools for protein domain analysis. Curr Protoc Bioinformatics 2008; Chapter 2:Unit 2.7.

21 Aoki-Kinoshita KF, Kanehisa M: Gene annotation and pathway mapping in KEGG. Methods Mol Biol 2007;396:71-91.

22 Zhang B, Kirov S, Snoddy J: WebGestalt: an integrated system for exploring gene sets in various biological contexts. Nucleic Acids Res 2005;33:W741-W748.

23 Kohl M, Wiese S, Warscheid B: Cytoscape: software for visualization and analysis of biological networks. Methods Mol Biol 2011;696:291-303.

-24 Von Mering C, Huynen M, Jaeggi D, Schmidt S, Bork P, Snel B: STRING: a database of predicted functional associations between proteins. Nucleic Acids Res 2003;31:258-261.

-25 Nepusz T, Yu H, Paccanaro A: Detecting overlapping protein complexes in protein-protein interaction networks. Nat Methods 2012;9:471-472.

26 Li K, Du X, He Y, Zhao L, Yang J, Song R, Chen L: P53-Rb signaling pathway is involved in tubular cell senescence in renal ischemia/reperfusion injury. Biocell 2007;31:213-223.

27 Alves MG, Machado NG, Sardao VA, Carvalho RA, Oliveira PJ: Anti-apoptotic protection afforded by cardioplegic celsior and histidine buffer solutions to hearts subjected to ischemia and ischemia/ reperfusion. J Cell Biochem 2011;112:3872-3881.

28 Banz Y, Rieben R, Zobrist C, Meier P, Shaw S, Lanz J, Carrel T, Berdat P: Addition of dextran sulfate to blood cardioplegia attenuates reperfusion injury in a porcine model of cardiopulmonary bypass. Eur J Cardiothorac Surg 2008;34:653-660.

29 Ouyang YB, Xu L, Lu Y, Sun X, Yue S, Xiong XX, Giffard RG: Astrocyte-enriched miR-29a targets PUMA and reduces neuronal vulnerability to forebrain ischemia. Glia 2013;61:1784-1794.

-30 Ziu M, Fletcher L, Rana S, Jimenez DF, Digicaylioglu M: Temporal differences in microRNA expression patterns in astrocytes and neurons after ischemic injury. PloS one 2011;6:e14724.

-31 Sengupta S, den Boon JA, Chen I-H, Newton MA, Stanhope SA, Cheng Y-J, Chen C-J, Hildesheim A, Sugden B, Ahlquist P: MicroRNA 29c is down-regulated in nasopharyngeal carcinomas, up-regulating mRNAs encoding extracellular matrix proteins. Proc Natl Acad Sci USA 2008;105:5874-5878.

32 Wang C-M, Wang Y, Fan C-G, Xu F-F, Sun W-S, Liu Y-G, Jia J-H: miR-29c targets TNFAIP3, inhibits cell proliferation and induces apoptosis in hepatitis B virus-related hepatocellular carcinoma. Biochem Biophys Res Commun 2011;411:586-592.

-33 Oren M: Decision making by p53: life, death and cancer. Cell Death Differ 2003;10:431-442.

-34 Weissmann N, Sydykov A, Kalwa H, Storch U, Fuchs B, y Schnitzler MM, Brandes RP, Grimminger F, Meissner M, Freichel M: Activation of TRPC6 channels is essential for lung ischaemia-reperfusion induced oedema in mice. Nat Commun 2012;3:649.

-35 Lin Y, Chen F, Zhang J, Wang T, Wei X, Wu J, Feng Y, Dai Z, Wu Q: Neuroprotective Effect of Resveratrol on Ischemia/Reperfusion Injury in Rats Through TRPC6/CREB Pathways. J Mol Neurosci 2013;1-10.

-36 Wang X, Teng L, Li A, Ge J, Laties AM, Zhang X: TRPC6 channel protects retinal ganglion cells in a rat model of retinal ischemia/reperfusion-induced cell death. Invest Ophthalmol Vis Sci 2010;51:5751-5758. 